\title{
New data on water beetles (Coleoptera: Gyrinidae, Haliplidae, Noteridae, Dytiscidae, Hydrophilidae, Elmidae) of Primorsky Krai (Russia)
}

\section{Новые данные по фауне водных жкуков (Coleoptera: Gyrinidae, Haliplidae, Noteridae, Dytiscidae, Hydrophilidae, Elmidae) Приморского края (Россия)}

\author{
A.S. Sazhnev ${ }^{1}$, A.A. Prokin ${ }^{1}$, M.E. Sergeev ${ }^{2}$ \\ А.С. Сажнев ${ }^{1}$, А.А. Прокин ${ }^{1}$, М.Е. Сергеев ${ }^{2}$
}

\footnotetext{
${ }^{1}$ Papanin Institute for Biology of Inland Waters, Russian Academy of Sciences, Borok 152742, Russia. E-mail: sazh@list.ru, prokina@mail.ru ${ }^{1}$ Институт биологии внутренних вод им. И.Д. Папанина Российской академии наук, Борок 152742, Россия.

${ }^{2}$ Federal Scientific Center of the East Asia Terrestrial Biodiversity, Far Eastern Branch, Russian Academy of Sciences, Vladivostok 690022, Russia. E-mail: eksgauster@inbox.ru

2 Федеральный научный центр наземной биоты Восточной Азии, Дальневосточное отделение Российской академии наук, Владивосток 690022, Россия.
}

KEY WORDS: Russian Far East, Sikhote-Alin Nature Reserve, new records, protected areas.

КЛЮЧЕВЫЕ СЛОВА: Дальний Восток России, Сихотэ-Алинский заповедник, новые находки, особо охраняемые природные территории.

ABSTRACT. Twenty-seven species of six families are recorded from the Sikhote-Alin Nature Reserve: Gyrinidae (1 species), Haliplidae (2), Noteridae (1), Dytiscidae (13), Hydrophilidae (9), and Hydraenidae (1). Additional records are provided for 29 species from Primorsky Krai: Dytiscidae (8), Hydrophilidae (20), and Elmidae (1). Three species (Oreodytes mongolicus, Sphaeridium bipustulatum, and $S$. marginatum) are recorded for the first time from the Russian Far East. One species of Gyrinidae (Gyrinus sachalinensis), one species of Dytiscidae (Oreodytes mongolicus), and two species of Hydrophilidae (Sphaeridium bipustulatum and $S$. marginatum) are recorded from Primorsky Krai for the first time. A new synonymy is established: Cercyon terminatus $($ Marsham, 1802) $=$ Cercyon emar ginatus Baranowski, 1985 syn.n.

РЕЗЮМЕ. В Сихотэ-Алинском заповеднике зарегистрировано 27 видов из шести семейств: Gyrinidae (1), Haliplidae (2), Noteridae (1), Dytiscidae (13), Hydrophilidae (9) и Hydraenidae (1). Приведены дополнительные сведения о находках 29 видов в Приморском крае: Dytiscidae (8), Hydrophilidae (20) и Elmidae (1). Три вида (Oreodytes mongolicus, Sphaeridium bipustulatum и S. marginatum) впервые указаны с Дальнего Востока России. Один вид Gyrinidae (Gyrinus sachalinensis), один вид Dytiscidae (Oreodytes mongolicus) и два вида Hydrophilidae
(Sphaeridium bipustulatum и S. marginatum) впервые отмечены в Приморском крае. Установлена новая синонимия: Cercyon terminatus (Marsham, 1802) = Cercyon emarginatus Baranowski, 1985 syn.n.

\section{Introduction}

The protected areas of Primorsky Krai in the Russian Far East include six nature reserves, four national parks, ten habitat/species management areas, one botanical garden, one dendrological park, and one nature park [Maslovskaia et al., 2020]. Our study is based mainly on material collected in the Sikhote-Alin Nature Reserve.

The Sikhote-Alin Nature Reserve is a biosphere reserve in Primorsky Krai. It is located in a watershed (between Sereryanka and Dzhigitovka Rivers) that stretches from the eastern part of the krai (province) of the humid golets-forested medium height eastern slopes of the Central Sikhote-Alin mountain range (Terneysky, Krasnoarmeysky, and Dalnegorski Districts of Primorsky Krai) up to the Sea of Japan. The total area of the Sikhote-Alin Nature Reserve is $3,901.84 \mathrm{~km}^{2}$.

The Sikhote-Alin Nature Reserve occupies the middle and lower reaches of the Kolumbe River (tributary of the Bolshaya Ussurka River), upper and middle reaches of the rivers Serebryanka and Zab-

How to cite this article: Sazhnev A.S., Prokin A.A., Sergeev M.E. 2021. New data on water beetles (Coleoptera: Gyrinidae, Haliplidae, Noteridae, Dytiscidae, Hydrophilidae, Elmidae) of Primorsky Krai (Russia) // Russian Entomol. J. Vol.30. No.3. P.264-274. doi: 10.15298/rusentj.30.3.03 
olochennaya, and three tributaries of the Dzhigitovka River (Kuruma, Lianovaya, and Kunaleyka Rivers) [Pimenova, 2016].

The Sikhote-Alin mountain range contains one the richest (in terms of the number of species) temperate forests of the world, located in the mixed forest zone
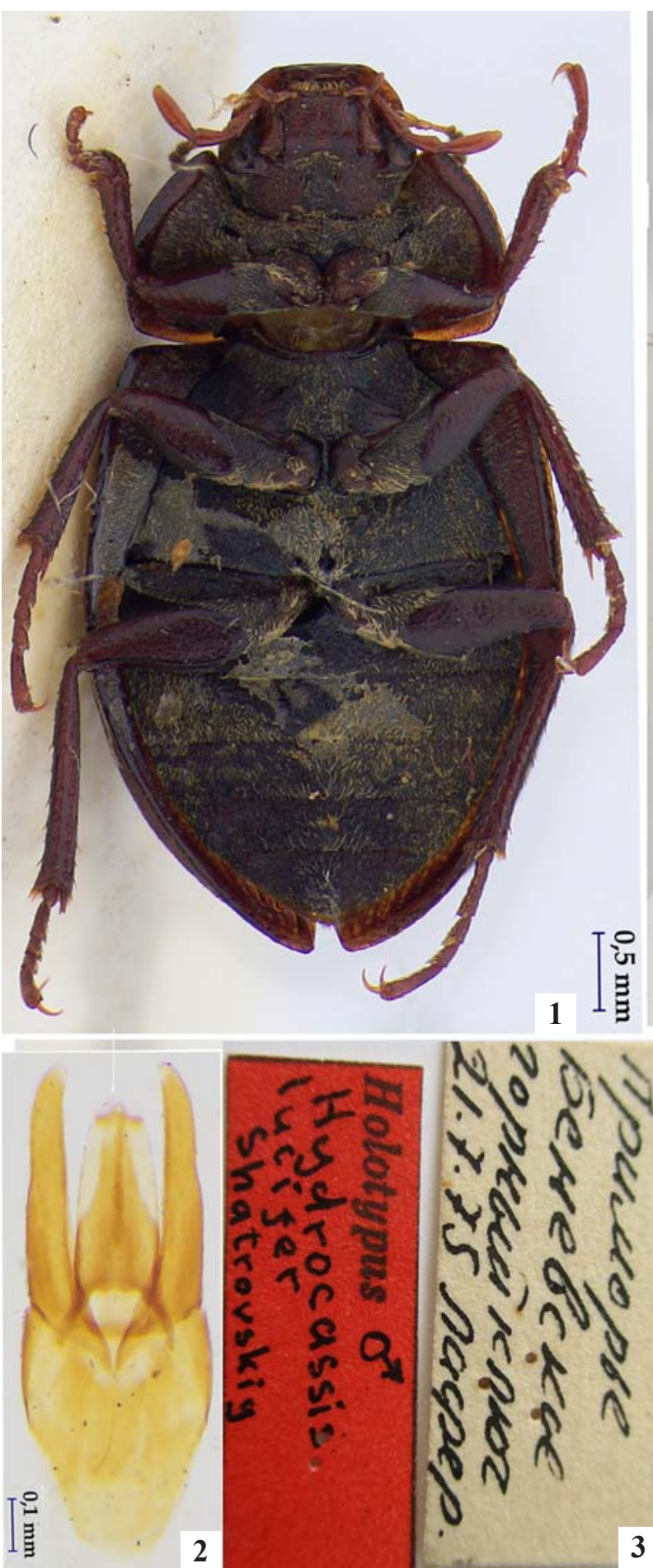

between the taiga and subtropics (this territory includes the distinct Okhotsk taiga oceanic and Manchurian moderate continental assemblages of broad-leaved forests). The climatic conditions of the Central Sikhote-Alin are defined by the regularities of the typical monsoon climate of Primorsky Krai.
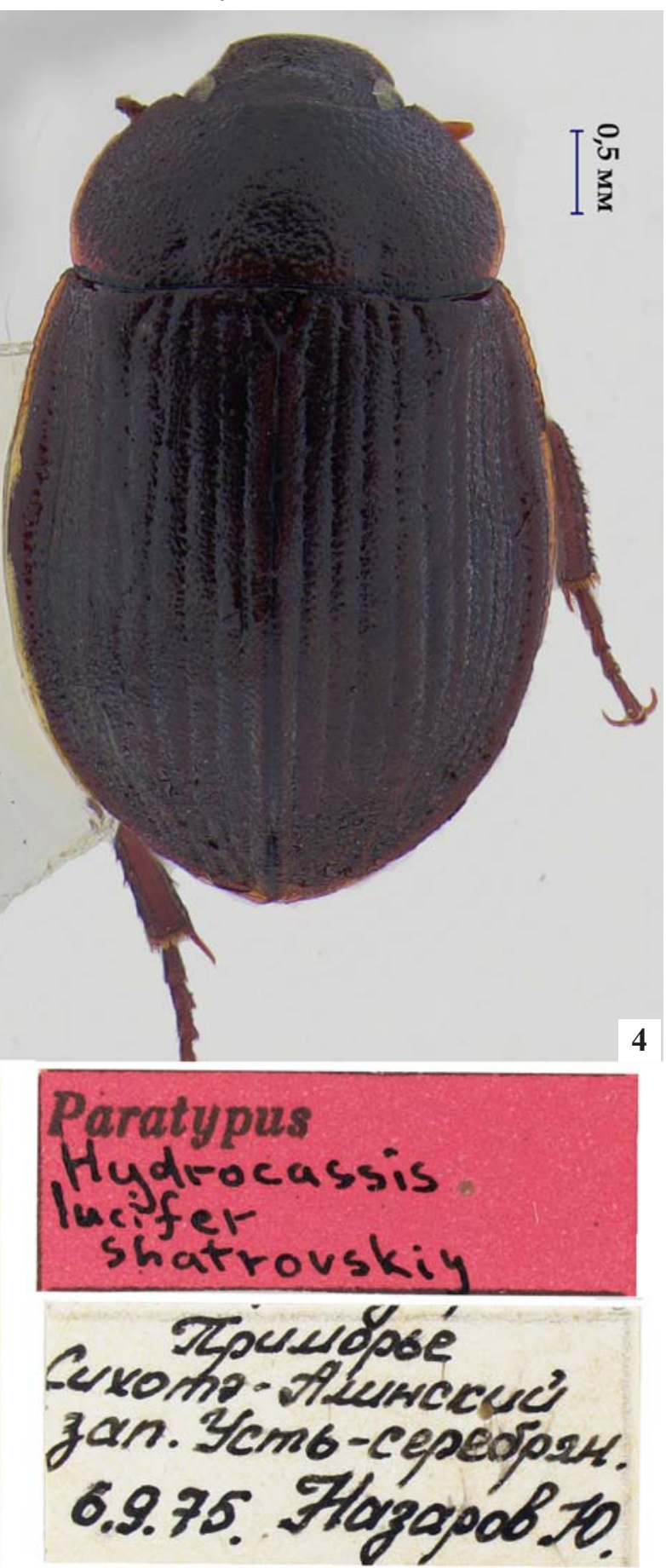

Figs 1-5. Ametor scabrosus Horn, 1873, holotype and paratype of Hydrocassis lucifer Shatrovskiy, 1989: 1 - habitus, ventral view; 2 - aedeagus; 3, 5 - labels; 4 - habitus, dorsal view; 1-3 - holotype; 4-5 - paratype. Photographs by V.M. Loktionov.

Pис. 1-5. Ametor scabrosus Horn, 1873; голотип и паратип Hydrocassis lucifer Shatrovskiy, 1989: 1 — габитус, снизу; 2 — эдеагус; 3, 5 - этикетки; 4 - габитус, сверху; 1-3 - голотип, 4-5 - паратип. Фотографии В.М. Локтионова. 
Unfortunately, published lists of the water beetles of the Sikhote-Alin Nature Reserve are sporadic and fragmentary, which determined the aim of our study. Only two species from studied families have been described from this territory: Hydrocassis lucifer Shatrovskiy, 1989 (Figs 1-5) - junior synonym of the Holarctic Ametor scabrosus Horn, 1873 (Hydrophilidae) — and Agabus (Gaurodytes) udege Nilsson, 1994 (Dytiscidae) [Shatrovskiy, 1989b, 1992; Nilsson, 1994].

In this study, we provide new records of beetles of the studied families from the Sikhote-Alin Nature Reserve and some other areas of the Primorsky Krai.

\section{Material and methods}

This study is mainly based on specimens collected by M.E. Sergeev (the material on the list is indicated without the name of the collector), which are deposited in the Papanin Institute for Biology of Inland Waters, Russian Academy of Sciences (Borok, Russia). The material was collected mainly by sweeping with an aquatic net or using a light trap.

The natural landmarks in the Sikhote-Alin Nature Reserve are represented by traditionally allocated areas of its territory [Pimenova, 2016]. The material was collected in 18 localities of the nature reserve (Fig. 6): 1 - Terney: floodplain of the Serebryanka River $\left(45.0335^{\circ}\right.$ N $\left.136.3733^{\circ} \mathrm{E}\right)$

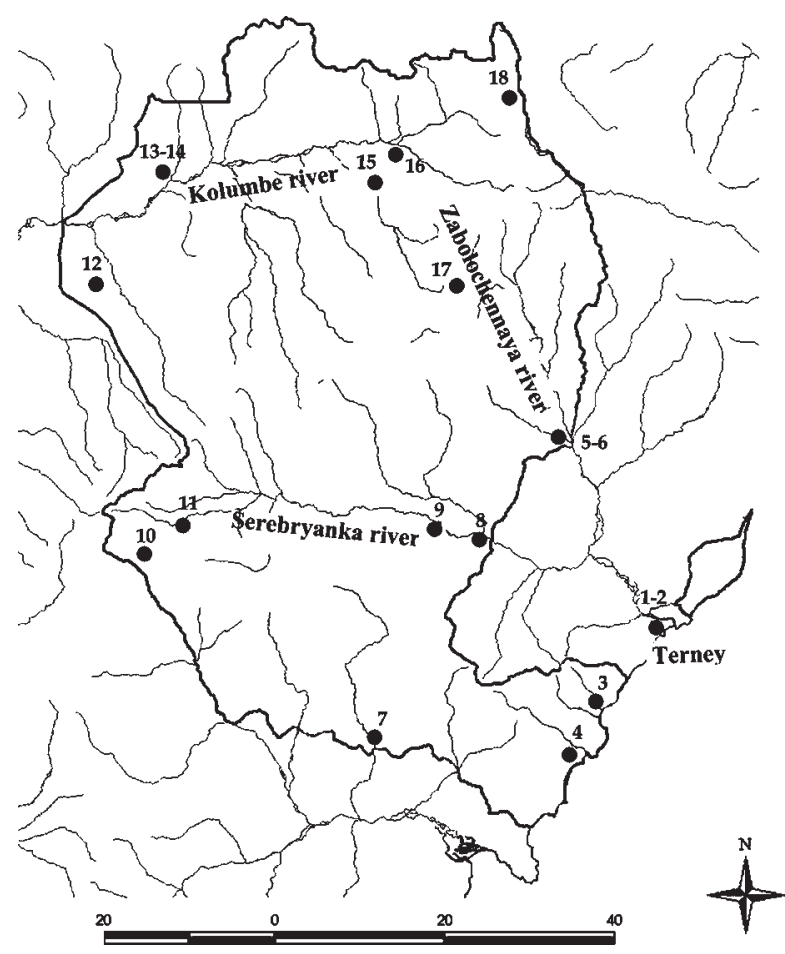

Fig. 6. Localities in the Sikhote-Alin Nature Reserve (numbers as in the text).

Рис. 6. Локалитеты на территории Сихотэ-Алинского заповедника (нумерация как в тексте).
2 - Terney: Ivan Ivanovicha branch of the Serebryanka River $\left(45.0833^{\circ} \mathrm{N} 136.6166^{\circ} \mathrm{E}\right)$;

3 - Blagodatnoe: vicinity of Blagodatnoe Lake $\left(44.5714^{\circ} \mathrm{N}\right.$ $\left.136.3250^{\circ} \mathrm{E}\right)$;

4 - Golubichnoe: vicinity of Golubichnoe Lake $\left(44.5430^{\circ} \mathrm{N}\right.$ $\left.136.3136^{\circ} \mathrm{E}\right)$;

5 - Yasnaya: floodplain of the Zabolochennaya River $\left(44.5325^{\circ} \mathrm{N} 136.2018^{\circ} \mathrm{E}\right)$;

6 - Yasnaya: floodplain of the Berezovy Stream $\left(45.2333^{\circ}\right.$ N $\left.136.5166^{\circ} \mathrm{E}\right)$;

7 - Kuruma: floodplain of the Kuruma River $\left(44.9152^{\circ} \mathrm{N}\right.$ $\left.136.2118^{\circ} \mathrm{E}\right)$;

8 - Ust-Serebryany: floodplain of the Serebryany Stream $\left(45.0825^{\circ} \mathrm{N} 136.2243^{\circ} \mathrm{E}\right)$;

9 - Zimoveyny: floodplain of the Zymoveyny Stream $\left(45.0829^{\circ} \mathrm{N} 136.1833^{\circ} \mathrm{E}\right)$;

10 - Kabany: floodplain of the Kabany Stream $\left(45.1102^{\circ} \mathrm{N}\right.$ $\left.135.8672^{\circ} \mathrm{E}\right)$;

11 - Sporny: upper reaches of the Serebryanka River $\left(45.1940^{\circ} \mathrm{N} 135.9901^{\circ} \mathrm{E}\right)$;

12 - Venera: floodplain of the Venera Stream $\left(45.2962^{\circ} \mathrm{N}\right.$ $\left.135.8010^{\circ} \mathrm{E}\right)$;

13 - Yupiter: floodplain of the Yupiter Stream $\left(45.5765^{\circ} \mathrm{N}\right.$ $\left.135.8928^{\circ} \mathrm{E}\right)$

14 - Kolumbe: floodplain of the Kolumbe River $\left(45.5387^{\circ}\right.$ $\mathrm{N} 135.9855^{\circ} \mathrm{E}$ );

15 - Rezvushka: floodplain of the Rezvushka River $\left(45.5410^{\circ}\right.$ N $136.1660^{\circ} \mathrm{E}$ );

16 - Ust-Prokhodnaya: stream in valley of the Kolumbe River $\left(45.3324 \mathrm{~N} 136.1365^{\circ} \mathrm{E}\right)$;

17 - Sakhalinsky: upper reaches of the Sakhalinsky Stream $\left(45.4147^{\circ} \mathrm{N} 136.3713^{\circ} \mathrm{E}\right)$;

18 - Perevalnaya: floodplain of the Tayozhnaya River $\left(45.6063^{\circ} \mathrm{N} 136.4454^{\circ} \mathrm{E}\right)$.

Photographs were taken with an Olympus SZX16 stereomicroscope and an Olympus DP74 digital camera and then stacked using Helicon Focus software. All scale bars are given in $\mathrm{mm}$. The illustrations were postprocessed for contrast and brightness corrections using Adobe Photoshop software.

\section{Results}

The results of this study are given as a list of species with information on the localities. The species list is based on the material studied. The species distribution data is mainly based on the Catalogue of Palaearctic Coleoptera [Fikáček et al., 2015; Hájek, 2017a, b; Hájek, Fery, 2017; Jäch, 2015; Jäch et al., 2016; Kobayashi et al., 2021; van Vondel, 2017]. New records from Primorsky Krai are indicated with an asterisk (*).

\section{List of species}

Family Gyrinidae Latreille, 1810

*Gyrinus (Gyrinus) sachalinensis Kamyia, 1936 Figs 7-10.

MATERIAL EXAMINED. 3:11.VI.2015, 3 exs., same locality, 24.VII.2017, 2 exs.; 8: 30.06.2018, 2 exs.

DISTRIBUTION. Russian Far East (Kuril Islands, Sakhalin), Japan [Nilsson et al., 2001; Hájek, Fery, 2017].

NOTE. The first record from continental Russian Far East and Primorsky Krai. 
Family Haliplidae Aubé, 1836

Haliplus (Haliplus) simplex Clark, 1863 kin).

MATERIAL EXAMINED. 12: 5.IX.2017, 3 exs. (G.A. Nachar-

DISTRIBUTION. Mongolia, North and South Korea, China, Russian Far East (Amur Oblast, Khabarovsky Krai and Primorsky Krai) [Zaitzev, 1953; Gramma, 1980; Lafer, 1996; Nilsson, Sundukov, 2009b; van Vondel, 2017].

Haliplus (Liaphlus) basinotatus A. Zimmermann, 1924

MATERIAL EXAMINED. 4: 17.VII.2017, 2 exs.

DISTRIBUTION. Mongolia, North and South Korea, north-eastern China, Russian Far East (Primorsky Krai, Sakhalin) [Nilsson, Sundukov, 2009b; van Vondel, 2017].

Family Noteridae C.G. Thomson, 1860

Noterus (Noterus) japonicus Sharp, 1873

MATERIAL EXAMINED. 9: 17.V.2017, 1 ex.

DISTRIBUTION. North-East China, "Korea," Russian Far East (Primorsky Krai), Japan [Nilsson, Sundukov, 2009c; Hájek, 2017b].

Family Dytiscidae Leach, 1815

Agabus (Acatodes) congener Thunberg, 1794

MATERIAL EXAMINED. 12: 2.VII.2019, 1 ex.

DISTRIBUTION. Europe, Asian Turkey, Kazakhstan, Mongolia, China (Jilin, Qinghai), West and East Siberia, Russian Far East (Amur Oblast, Khabarovsky Krai, Kuril
Islands, Magadan Oblast, Primorsky Krai, Sakhalin), Japan [Lafer, 1989; Nilsson, Kholin, 1994; Nilsson et al., 1999; Nilsson, Sundukov, 2009a; Hájek, 2017a].

Agabus (Gaurodytes) affinis (Paykull, 1798)

MATERIAL EXAMINED. 4: 18.IX.2018, 1 ex.

DISTRIBUTION. Europe, West and East Siberia, Russian Far East (Kamchatka, Primorsky Krai), Japan (Hokkaido) [Zaitzev, 1953; Lafer, 1989; Nilsson, Sundukov, 2009a; Hájek, 2017a].

Agabus (Gaurodytes) udege Nilsson, 1994 4 exs.

MATERIAL EXAMINED. 1: 6.VIII.2015, 1 ex.; 11: 1.VIII.2020,

DISTRIBUTION. China (Jilin), Mongolia, North Korea, Russian Far East (Primorsky Krai) [Nilsson, 1994; Nilsson, Sundukov, 2009a; Hájek, 2017a; Enkhnasan, Boldgiv, 2019].

Ilybius anjae Nilsson, 1999

MATERIAL EXAMINED. 3: 7.IX.2016, 1 ex.

DISTRIBUTION. East Siberia, China (Heilongjiang), Russian Far East (Amur Oblast, Khabarovsky and Primorsky krai, Sakhalin), Japan [Nilsson, 1999; Hájek, 2017a].

Ilybius chishimanus Kôno, 1944

MATERIAL EXAMINED. 12: 2.VII.2019, 1 ex.

DISTRIBUTION. East Siberia, Mongolia, China (Heilongjiang, Jilin), Russian Far East (Kamchatka, Kuril Islands, Primorsky Krai) [Nilsson et al., 1997, 1999; Nilsson, Sundukov, 2009a; Hájek, 2017a].
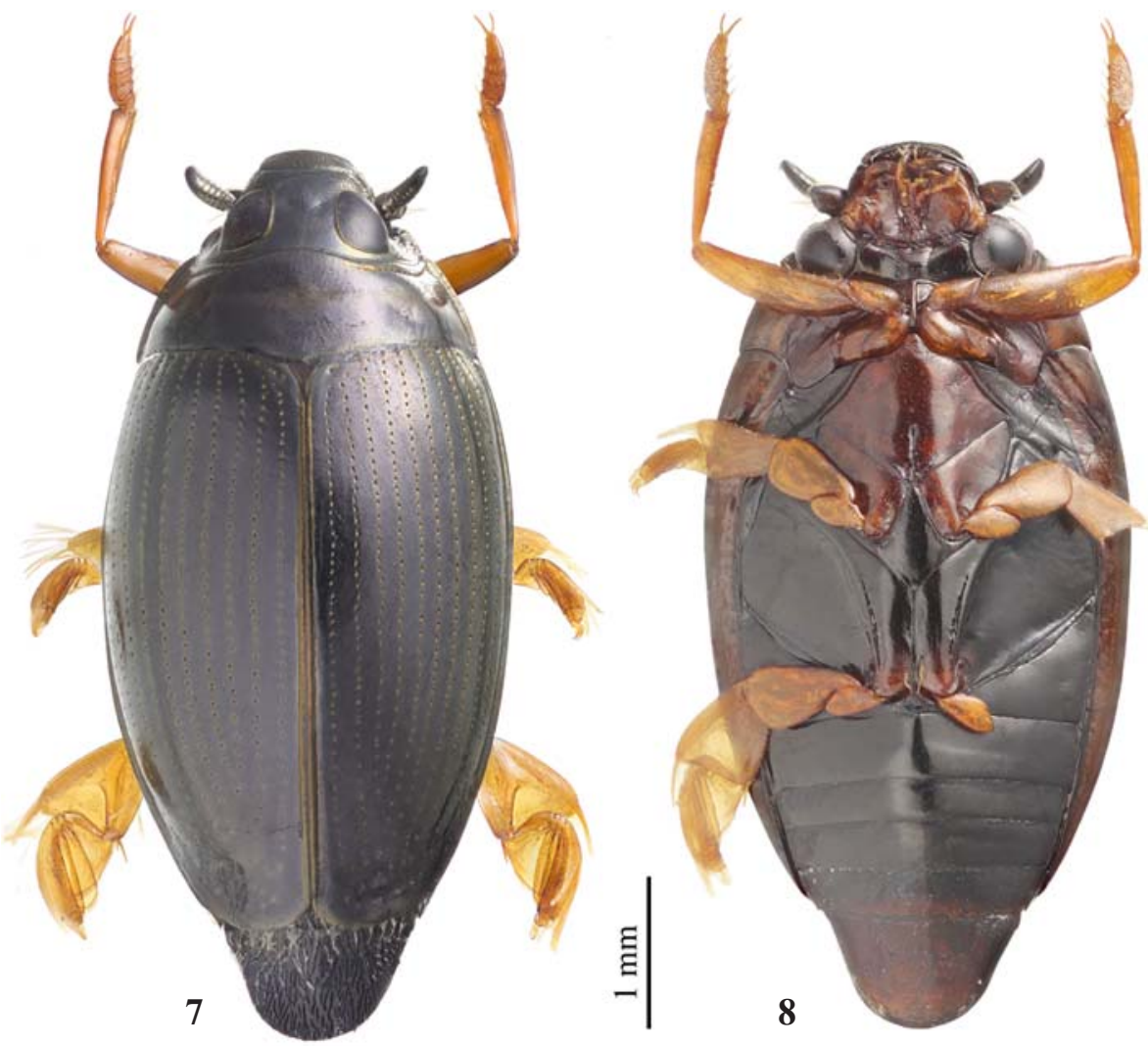

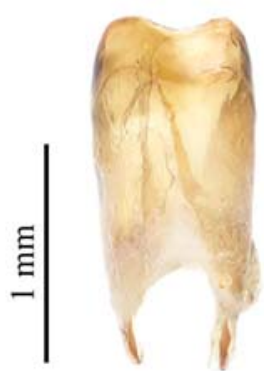

9

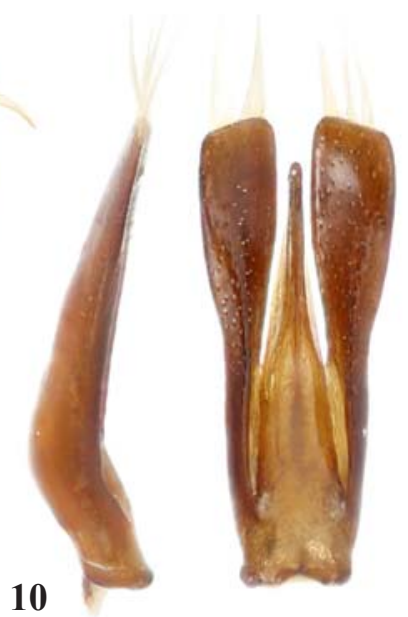

Figs 7-10. Gyrinus sachalinensis Kamyia, 1936 from floodplain of the Serebryany Stream: 7-8 - habitus, dorsal (7) and ventral (8) view; 9 - ninth sternite; 10 - male genitalia, lateral and dorsal view. Photographs by K.V. Makarov.

Рис. 7-10. Gyrinus sachalinensis Kamyia, 1936 из поймы Серебряного ручья: 7-8 — габитус, сверху (7) и снизу (8); 9 - девятый стернит; 10 - гениталии самца, сбоку и сверху. Фотографии К.В. Макарова. 
*Ilybius opacus Aubé, 1837

MATERIAL EXAMINED. 15: 20.IV.2018, 2 exs.; 16: 13.VI.2016, 1 ex.

DISTRIBUTION. Northern Europe, Mongolia, West and East Siberia, Russian Far East (Kamchatka, Sakhalin, Primorsky Krai) [Nilsson et al., 1999; Hájek, 2017a], North America [Larson et al., 2000].

Platambus koreanus (Nilsson, 1997)

MATERIAL EXAMINED. 4: 29.VI.2016, 1 ex.

DISTRIBUTION. South Korea, Russian Far East (Primorsky Krai) [Nilsson, Sundukov, 2009a; Hájek, 2017a].

Acilius (Acilius) sulcatus (Linnaeus, 1758)

MATERIAL EXAMINED. 4: 30.IX.2015, 1 ex.

DISTRIBUTION. Europe, North Africa (Algeria), Iran, Kirgizstan, Kazakhstan, Turkmenistan, Mongolia, West and East Siberia, Russian Far East (Primorsky Krai, Sakhalin) [Zaitzev, 1953; Lafer, 1989; Nilsson, Kholin, 1994; Hájek, 2017a; Prokin et al., 2020].

Dytiscus dauricus dauricus Gebler, 1832

MATERIAL EXAMINED. 4: 29.IV.2018, 1 ex.; 13: 17.VII.2017, 1 ex.

DISTRIBUTION. Mongolia, East Siberia, China (Heilongjiang, Jilin, Xinjiang), Russian Far East (Kamchatka, Khabarovsky Krai, Kuril Islands, Magadan Oblast, Primorsky Krai, Sakhalin), Japan, North America [Zaitzev, 1953; Lafer, 1989; Nilsson, Kholin, 1994; Nilsson et al., 1997, 1999; Nilsson, Larson et al., 2000; Sundukov, 2009a; Hájek, 2017a].

Dytiscus marginalis czerskii Zaitzev, 1953

MATERIAL EXAMINED. 1: 12.IV.2017, 1 ex.

DISTRIBUTION. Asian subspecies distributed in East Siberia, North Korea, north-eastern China, Russian Far East (Kuril Islands, Primorsky Krai, Sakhalin,), Japan [Zaitzev, 1953; Lafer, 1989; Nilsson, Sundukov, 2009a; Hájek, 2017a].

*Oreodytes mongolicus Brinck, 1943

MATERIAL EXAMINED. 7: 5-10.VI.2020, 2 exs.

DISTRIBUTION. Tuva, Mongolia [Brinck, 1943; Prokin et al., 2020]. Krai.

NOTES. The first record from Far East and Primorsky

Oreodytes okulovi Lafer, 1988

MATERIAL EXAMINED. 8: 30.VI.2018, 1 ex.

DISTRIBUTION. East Siberia, Russian Far East (Jewish Autonomous Oblast, Kamchatka, Primorsky Krai) [Lafer, 1988; Nilsson et al., 1999; Fery, Petrov, 2014; Hájek, 2017a].

Hydroporus uenoi Nakane, 1963 1 ex.

MATERIAL EXAMINED. 5: 4.VII.2015, 1 ex.; 16: 17.V.2018,

DISTRIBUTION. East Siberia, Mongolia, China (Heilongjiang, Jilin), Russian Far East (Kamchatka, Khabarovsky Krai, Kuril Islands, Primorsky Krai, Sakhalin), Japan [Nilsson, Kholin, 1994; Nilsson et al., 1997, 1999; Hájek, 2017a].

Family Hydrophilidae Latreille, 1802

Hydrobius fuscipes (Linnaeus, 1758) sensu lato

MATERIAL EXAMINED. 4: 18.IX.2018, 1 ex.; 7: 20.IV.2018, 2 ex.; 15: 28.V.2020, 1 ex.; 16: 17.V.2018, 1 ex.; 18: 20.IV.2018, 2 ex.

DISTRIBUTION. Europe, North Caucasus, Azerbaijan, Near East, Central Asia, West and East Siberia, Mongolia, China, Russian Far East (Amur Oblast, Khabarovsky Krai, Kuril Islands, Magadan Oblast, Primorsky Krai); North Amer- ica [Shatrovskiy, 1989b; Hebauer, 1995; Prokin, 2009; Fikáček et al., 2015; Ryndevich, 2016; Ryndevich, Angus, 2020].

NOTE. The Holarctic morphospecies Hydrobius fuscipes (Linnaeus, 1758) is represented by a complex of cryptic species, recently partly revised [Fossen et al., 2016; Ryndevich, Angus, 2020]. Material from the Far East needs to be revised using cytogenetic and molecular methods.

Hydrochara affinis Sharp, 1873

MATERIAL EXAMINED. 1: 23.VII.2016, 1 ex.; 3: 10.VIII.2016, 1 ex.

DISTRIBUTION. South European territory of Russia, North Caucasus, Azerbaijan, Central Asia, China, Mongolia, East Siberia, South Korea, Russian Far East (Amur Oblast, Khabarovsky Krai and Primorsky Krai, Sakhalin), Japan [Smetana, 1980; Shatrovskiy, 1986, 1989b; Hebauer, 1995; Hebauer, Ryndevich, 2005; Prokin, 2009; Fikáček et al., 2015].

\section{Crenitis (Crenitis) apicalis (Reitter, 1896)}

MATERIAL EXAMINED. 1: 21.IV.2015, 5 exs. (D.E. Shcherbakov); 2: 21.IV.2015, 1 ex. (D.E. Shcherbakov); 3: 21.VI.2016, 2 exs.; 5: 4.VII.2015, 9 exs.; 6: 14-23.IV.2015, 5 ex. (D.E. Shcherbakov); 7: 5-10.VI.2020, 5 ex.; 8: 30.VI.2018, 6 exs.; 9: 18.IV.2018, 4 ex.; 10: 24-27.IV.2015, 20 exs. (D.E. Shcherbakov), same place, 26-28.V.2015, 8 exs.; 11: 1.VIII.2020, 5 exs.; 16: 17.V.2018, 6 exs.; 17: 30.V.2020, 12 exs.; 18: 20.IV.2018, 11 exs.

DISTRIBUTION. East Siberia, Mongolia, north-eastern China, Russian Far East (Amur Oblast, Khabarovsky Krai, Magadan Oblast, Primorsky Krai), Japan [Shatrovskiy, 1989b; Hebauer, 1994, 1995; Prokin, 2009; Fikáček et al., 2015; Prokin et al., 2020].

Cercyon (Cercyon) aptus Sharp, 1873

MATERIAL EXAMINED. 4: 15.VII.2017, 3 exs.

DISTRIBUTION. China (Hebei), Russian Far East (Kuril Islands, Primorsky Krai, Sakhalin), Japan [Shatrovskij, 1992; Hebauer, 1995; Ryndevich, 2003; Ôhara, Jia, 2006; Prokin, 2009; Fikáček et al., 2015].

Cercyon (Cercyon) numerosus Shatrovskiy, 1989

MATERIAL EXAMINED. 1: 8.VIII.2018, 3 exs.; 3: 1.VI.2018, 7 exs.; 4: 5.VII.2019, 1 ex.

DISTRIBUTION. South Korea, Russian Far East (Kuril Islands, Primorsky Krai), Japan [Shatrovskij, 1992; Hebauer, 1995; Ryndevich, 2001b; Ôhara, Jia, 2006; Prokin, 2009; Fikáèek et al., 2015].

Cercyon (Cercyon) olibrus Sharp, 1874

MATERIAL EXAMINED. 5: 11.VII.2018, 1 ex.

DISTRIBUTION. China (Jiangxi, Tianjin), Russian Far East (Amur Oblast, Khabarovsky Krai, Kuril Islands, Primorsky Krai), Japan [Shatrovskiy, 1989b; Hebauer, 1995; Ôhara, Jia, 2006; Prokin, 2010; Fikáček et al., 2015].

Cercyon (Cercyon) symbion Shatrovskiy, 1989

MATERIAL EXAMINED. 3: 1.VI.2018, 1 ex.; 4: 5.VII.2019, 1 ex. DISTRIBUTION. Russian Far East (Commander Islands, Kuril Islands, Primorsky Krai, Sakhalin, Isl.), Japan [Shatrovskiy, 1989b, 1992; Prokin, 2009; Fikáček et al., 2015; Sazhnev, 2018].

\section{Cercyon (Cercyon) terminatus (Marsham, 1802)} Figs 11-14.

= Cercyon (Cercyon) emarginatus Baranowski, 1985, syn. nov. MATERIAL EXAMINED. 5: 11.VII.2018, 1 ex.; 15: 28.V.2020, 2 exs.; 16: 18.VI.2015, 5 exs.

DISTRIBUTION. Europe, North Africa, Near East, Kazakhstan, West Siberia, Mongolia, China (Shaanxi), Russian 
Far East (Amur Oblast, Khabarovsky Krai and Primorsky Krai) [Shatrovskiy, 1989b; Fikáček et al., 2015], introduced to North America [Smetana, 1978, 1988].

NOTE. Studied specimens from Primorsky Krai morphologically are identical to Cercyon emarginatus Baranowski, 1985 description, but the external characters of this species are within the variability range of Cercyon terminatus (Marsham, 1802); the structure of the genitalia according to Baranowski [1985: P. 343, Figs 1-6] shows that type series is identical to that of specimens of $C$. terminatus from European Russia and Primorsky Krai (Fig. 4), as well as with pictures provided by A. Smetana for North American specimens [Smetana, 1978: Figs 133-135; Smetana, 1988: Figs 345347]. Thus, a new synonymy is established here.

\section{Cercyon (Clinocercyon) retius Ryndevich et Prokin,} 2017

MATERIAL EXAMINED. 10: 28.05.2015, 2 exs.

DISTRIBUTION. Russian Far East (Kunashir Island, Primorsky Krai) [Ryndevich, Prokin, 2017].

Family Hydraenidae Mulsant, 1844

Hydraena (Hydraena) riparia Kugelann, 1794

MATERIAL EXAMINED. 1: 21.IV.2015, 4 exs. (D.E. Shcherbakov); 2: 21.IV.2015, 8 exs. (D.E. Shcherbakov); 5: 14-23.04.2015,

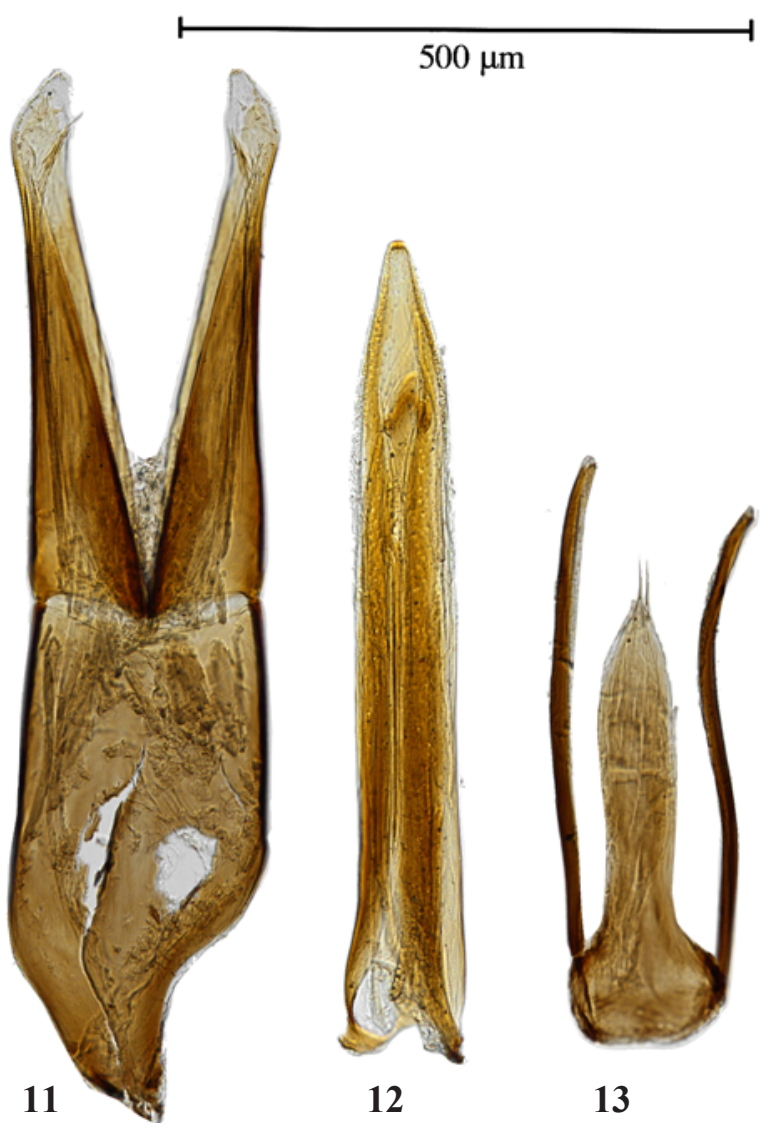

Figs 11-13. Male genitalia of Cercyon terminatus (Marsham, 1802) from the Sikhote-Alin Nature Reserve: 11 - parameres (lateral lobes) and phallobase; 12 - penis (median lobe); 13 - ninth ventrite. Photographs by A.A. Prokin.

Рис. 11-13 Гениталии самца Cercyon terminatus (Marsham, 1802) из Сихотэ-Алинского заповедника: 11 - парамеры и фаллобаза; 12 - пенис; 13 - девятый вентрит. Фотографии А.А. Прокина.
21 exs. (D.E. Shcherbakov); 10: 24-27.IV.2015, 8 exs. (D.E. Shcherbakov).

DISTRIBUTION. Europe, Central Asia, Mongolia, West and East Siberia, China, South Korea, Russian Far East (Kuril Islands, Primorsky Krai), Japan [Jäch, 1988, 2015; Shatrovskiy, 1989a, Gusakov, 2009; Palatov, 2014].

The studied material from the Sikhote-Alin Nature Reserve is represented by 27 species of six families: Gyrinidae (1 species), Haliplidae (2), Noteridae (1), Dytiscidae (13), Hydrophilidae (9), and Hydraenidae (1).

Taking into account published data, the fauna of the studied families of the Sikhote-Alin Nature Reserve includes only 28 recorded species, which indicates an insufficient level of regional faunal investigation. For example, more than 80 species of beetles of the studied families are recorded from the Lazovsky Nature Reserve [Gusakov, 2009; Nilsson, Sundukov, 2009a, b, c; Prokin, 2009, 2010].

\section{Additional records from Primorsky Krai}

Additional material was collected by different persons in 2015-2020 from various protected areas and their buffer zones in Primorsky Krai (Fig. 14). Distributional data for species that were indicated in the list above are not provided again.

\section{Family Dytiscidae Leach, 1815}

Ilybius chishimanus Kôno, 1944

MATERIAL EXAMINED. Krasnoarmeysky Distr., "Udygeyskaya Legenda" National Park, floodplain of Armu River, 23.VII.2015, 1 ex.

Rhantus (Rhantus) suturalis (W.S. Macleay, 1825)

MATERIAL EXAMINED. Khasansky Distr., Vityaz village, unnamed stream, 12.IX.2019, 1 ex.; Spassk-Dalny, light-trapped, 10.VII.2016, 1 ex.; Peter the Great Bay, Popova Island, Starka village, light-trapped, 27.VIII.2018, 1 ex.; Vladivostok, Okeanskaya station, 24.09.2020, 1 ex.

DISTRIBUTION. Europe, North Africa, Near East, Central Asia, West and East Siberia, China, Mongolia, Russian Far East (Kuril Islands, Primorsky Krai, Sakhalin), Japan; North America, Australian and Oriental regions [Zaitzev, 1953; Lafer, 1989; Nilsson et al., 1997, 1999; Hájek, 2017a].

Copelatus weymarni J. Balfour-Browne, 1947

MATERIAL EXAMINED. Spassk-Dalny, light-trapped, 10.VII.2016, 2 exs.

DISTRIBUTION. north-eastern China, North and South Korea, Russian Far East (Amur Oblast, Primorsky Krai), Japan [Nilsson, Sundukov, 2009a; Hájek, 2017a].

Graphoderus adamsii (Clark, 1864)

MATERIAL EXAMINED. Spassk-Dalny, light-trapped, 10.VII.2016, 1 ex.

DISTRIBUTION. China, South Korea, Russian Far East (Primorsky Krai), Japan [Zaitzev, 1953; Lafer, 1989; Hájek, 2017a].

\section{Hydaticus (Prodaticus) grammicus (Germar, 1827)}

MATERIAL EXAMINED. Spassk-Dalny, light-trapped, 10.VII.2016, 1 ex.

DISTRIBUTION. Europe, Near East, Central Asia, China, North Korea, Japan (Hájek, 2017a); Russian Far East (Amur Oblast, Primorsky Krai) [Zaitzev, 1953; Lafer, 1992; Berlov, Berlov, 1996; Nilsson, Sundukov, 2009a].

*Oreodytes mongolicus Brinck, 1943

MATERIAL EXAMINED. Krasnoarmeysky Distr., Udygeyskaya Legenda National Park, floodplain of Armu River, 23.VII.2015, 1 ex. 
Hydroporus uenoi Nakane, 1963

MATERIAL EXAMINED. Spassk-Dalny, light-trapped, 10.VII.2016, 1 ex.

Hygrotus (Coelambus) chinensis (Sharp, 1882)

MATERIAL EXAMINED. Spassk-Dalny, light-trapped, 10.VII.2016, 2 exs.

DISTRIBUTION. Mongolia, China, North and South Korea, Russian Far East (Primorsky Krai), Japan [Nilsson, Sundukov, 2009a; Hájek, 2017a].

Family Hydrophilidae Latreille, 1802

Berosus (Berosus) punctipennis Harold, 1878

MATERIAL EXAMINED. Spassk-Dalny, light-trapped, 10.VII.2016, 5 exs.

DISTRIBUTION. Mongolia, East Siberia, China, South Korea, Russian Far East (Amur Oblast, Khabarovsky Krai and Primorsky Krai), Japan [Schödl, 1993; Hebauer, 1995; Ryndevich, Fikáček, 2013; Fikáček et al., 2015].

Berosus (Enoplurus) lewisius Sharp, 1873

MATERIAL EXAMINED. Chuguevsky Distr., Chuguevka, light-trapped, 1-3.IX.2020, 1 ex. (K.A. Ostapenko).

DISTRIBUTION. Mongolia, China, North and South Korea, Russian Far East (Khabarovsky Krai and Primorsky Krai); Oriental region [Shatrovskiy, 1989b; Schödl, 1991; Hebauer, 1995; Prokin, 2009; Ryndevich, 2001a, 2011; Fikáček et al., 2015].

Hydrobius fuscipes (Linnaeus, 1758) sensu lato

MATERIAL EXAMINED. Spassk-Dalny, light-trapped, 10.07.2016, 9 exs.; Vladivostok, Botanical Garden, 25.VIII.2019, 1 ex.; Peter the Great Bay, Russky Island, 1.X.2019, 1 ex.
Hydrochara affinis Sharp, 1873

MATERIAL EXAMINED. Spassk-Dalny, light-trapped, 10.VII.2016, 8 exs.

Hydrophilus (Hydrophilus) dauricus Mannerheim, 1852

MATERIAL EXAMINED. Spassk-Dalny, light-trapped, 10.VII.2016, 1 ex.

DISTRIBUTION. Mongolia, East Siberia, China, Russian Far East (Amur Oblast, Primorsky Krai), Japan [Shatrovskiy, 1989b; Prokin, 2009; Fikáček et al., 2015; Prokin et al., 2020].

Anacaena lutescens (Stephens, 1829)

MATERIAL EXAMINED. Chuguevsky District, Chuguevka, light-trapped, 1-3.IX.2020, 3 exs. (K.A. Ostapenko).

DISTRIBUTION. Europe, North Africa, Central Asia, West Siberia, China (Xinjiang), Russian Far East (Primorsky Krai); North America [Prokin, 2009; Fikáček et al., 2015].

Enochrus (Enochrus) melanocephalus (Olivier, 1792)

MATERIAL EXAMINED. Spassk-Dalny, light-trapped, 10.VII.2016, 1 ex.

DISTRIBUTION. Europe, North Africa, Near East, Central Asia, Afghanistan, Mongolia, China (Inner Mongolia), East Siberia, Russian Far East (Primorsky Krai) [Ryndevich, 2001a; Prokin, 2009; Fikáček et al., 2015; Prokin et al., 2020].

Enochrus (Holcophilydrus) simulans (Sharp, 1873)

MATERIAL EXAMINED. Spassk-Dalny, light-trapped, 10.VII.2016, 3 exs.

DISTRIBUTION. China, South Korea, Russian Far East (Primorsky Krai), Japan [Shatrovskiy, 1989b; Hebauer, 1995; Prokin, 2009; Fikáček et al., 2015].

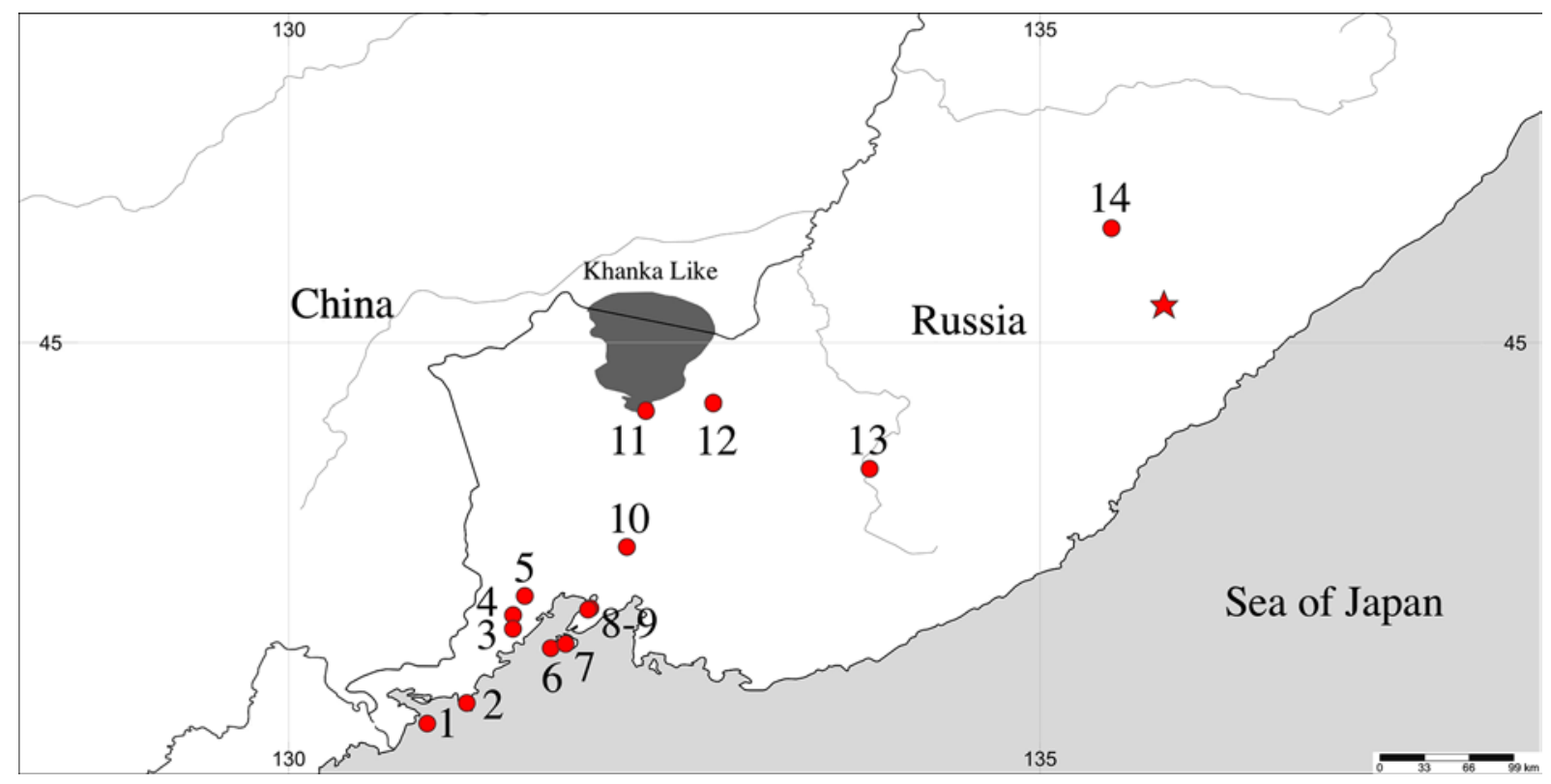

Fig. 14. Localities in Primorsky Krai: red star — The Sikhote-Alin Nature Reserve, red circles: 1 — Furugelm Island; 2 — Vityaz village; 3 — "Kedrovaya Pad" Nature Reserve; 4 - Barabash village; 5 — Zanadvorovka village; 6 — Popova Island; 7 — Russky Island; 8-9 Vladivostok, Okeanskaya station and Botanical Garden; 10 - Kaymanovka village; 11 - Khanka Nature Reserve, Sopka Luzanova; 12 Spassk-Dalny; 13 - Chuguevka village; 14 - Udygeyskaya Legenda National Park.

Рис. 14. Локалитеты на территории Приморского края. Красная звезда - Сихотэ-Алинский заповедник, красные круги: 1 остров Фуругельма; 2 - с. Витязь; 3 - заповедник «Кедровая падь»; 4 - с. Барабаш; 5 - с. Занадворовка.; 6 - остров Попова; 7 - остров Русский; 8-9 - Владивосток, ст. Океанская и ботанический сад; 10 - с. Каймановка; 11 - Ханкайский заповденик, "Сопка Лузанова"; 12 - Спасск-Дальний; 13 - с. Чугуевка; 14 - национальный парк "Удыгейская легенда". 
Enochrus (Lumetus) quadripunctatus (Herbst, 1797)

MATERIAL EXAMINED. Spassk-Dalny, light-trapped, 10.VII.2016, 1 ex.; Peter the Great Bay, Far East Marine Reserve, Furugelm Island, 17.VIII.2018, 1 ex.

DISTRIBUTION. Europe, Near East, Central Asia, Mongolia, China, West and East Siberia, Russian Far East (Amur Oblast, Khabarovsky Krai and Primorsky Krai) [Shatrovskiy, 1989b; Prokin, 2009; Ryndevich, Fikáček, 2013; Fikáček et al., 2015; Prokin et al., 2020].

Enochrus (Methydrus) affinis (Thunberg, 1794)

MATERIAL EXAMINED. Spassk-Dalny, light-trapped, 10.VII.2016, 3 exs.

DISTRIBUTION. Europe, Kazakhstan, Mongolia, China (Heilongjiang, Inner Mongolia), West and East Siberia, Russian Far East (Khabarovsky Krai and Primorsky Krai, Sakhalin), Japan [Shatrovskiy, 1989b; Hebauer, 1995; Prokin, 2009; Fikáček et al., 2015].

Enochrus (Methydrus) japonicus (Sharp, 1873) Figs 15-17.

MATERIAL EXAMINED. Spassk-Dalny, light-trapped, 10.VII.2016, 8 exs.
DISTRIBUTION. China, Russian Far East (Primorsky Krai), Japan; Oriental region [Shatrovskiy, 1989b; Hebauer, 1995; Fikáček et al., 2015].

Enochrus (Methydrus) puetzi Hebauer, 1995

MATERIAL EXAMINED. Spassk-Dalny, light-trapped, 10.VII.2016, 4 exs.

DISTRIBUTION. Russian Far East (Primorsky Krai) [Hebauer, 1995; Prokin, 2009; Fikáček et al., 2015].

Crenitis (Crenitis) apicalis (Reitter, 1896)

MATERIAL EXAMINED. Khasansky Distr., "Kedrovaya Pad" Nature Reserve, Barabash village, 10.05.2019, 1 ex.; Ussuriysk, Kaymanovka village, Barsukovka River, $43.6437^{\circ} \mathrm{N} 132.2504^{\circ} \mathrm{E}$, 18.06.2020, 11 exs. (I.A. Zabaluev).

Crenitis (Crenitis) kanyukovae Shatrovskiy, 1989

MATERIAL EXAMINED. Khasansky Distr., 2 km NW Zanadvorovka village, unnamed spring, $43.3172^{\circ} \mathrm{N} 131.5712^{\circ} \mathrm{E}, 235 \mathrm{~m}$ a.s.1., 10.VII.2020, 2 exs. (I.A. Zabaluev).

DISTRIBUTION. Russian Far East (Primorsky Krai) [Shatrovskiy, 1992; Hebauer, 1994, 1995; Prokin, 2009; Fikáček et al., 2015].

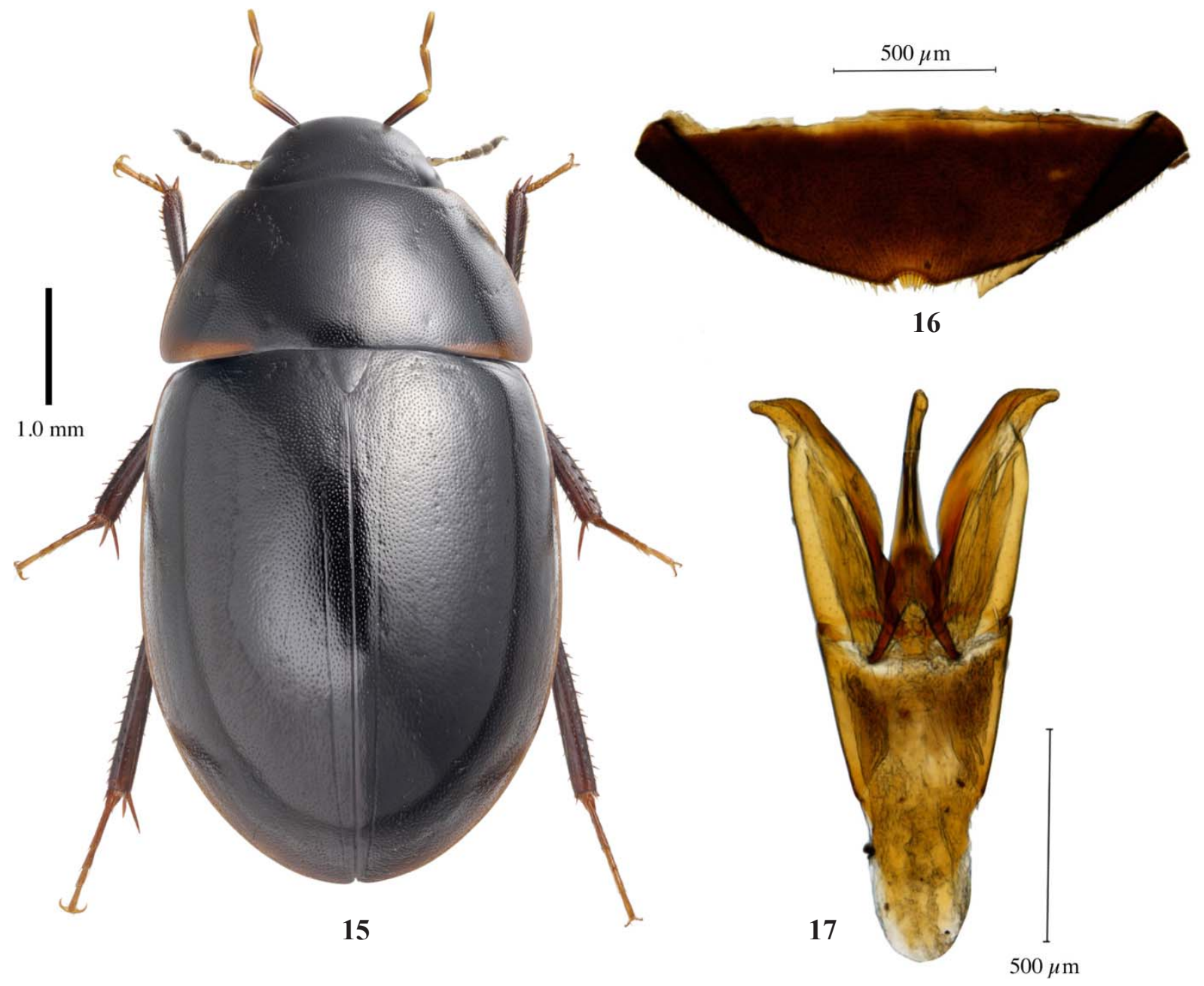

Figs 15-17. Enochrus japonicus (Sharp, 1873): 15 - habitus, dorsal view; 16 - fifth ventrite; 17 - aedeagus. Photographs by K.V. Makarov (15) and A.A. Prokin (16-17).

Рис. 15-17. Enochrus japonicus (Sharp, 1873): 15 - габитус, сверху; 16 - пятый вентрит; 17 - эдеагус. Фотографии К.В. Макарова (15) и А.А. Прокина (16-17). 
Coelostoma (Coelostoma) orbiculare (Fabricius, 1775)

MATERIAL EXAMINED. Khanka Nature Reserve, Sopka Luzanova landscape unit (natural boundary), 5.VII.2016, 1 ex. (M.E. Sergeev); Spassk-Dalny, light-trapped, 10.VII.2016, 11 exs.

DISTRIBUTION. Europe, Central Asia, China, East and West Siberia, Russian Far East (Primorsky Krai), Japan [Shatrovskiy, 1989b; Fikáček et al., 2015].

Cercyon (Cercyon) bifenestratus Küster, 1851

MATERIAL EXAMINED. Spassk-Dalny, light-trapped, 10.VII.2016, 1 ex.

DISTRIBUTION. Europe, Central Asia, Nepal, Mongolia, China, East and West Siberia, Russian Far East (Primorsky Krai), Japan [Shatrovskiy, 1989b; Hebauer, 1995; Fikáček et al., 2015].

Cercyon (Cercyon) marinus C.G. Thomson, 1853

MATERIAL EXAMINED. Spassk-Dalny, light-trapped, 10.VII.2016, 2 exs.

DISTRIBUTION. Europe, Kazakhstan, Mongolia, East and West Siberia, Russian Far East (Amur Oblast, Kamchatka, Khabarovsky Krai and Primorsky Krai) [Shatrovskiy, 1989b; Hebauer, 1995; Fikáček et al., 2015].

*Sphaeridium bipustulatum Fabricius, 1781

MATERIAL EXAMINED. Khanka Nature Reserve, landscape unit (natural boundary) "Sopka Luzanova", 6.VII.2016, 1 ex.

DISTRIBUTION. Widely distributed in Europe, North Africa, Near East, Central Asia, East and West Siberia; introduced to North America [Fikáček et al., 2015].

NOTE. The first record from Russian Far East and Primorsky Krai.

*Sphaeridium marginatum Fabricius, 1787

MATERIAL EXAMINED. Khanka Nature Reserve, landscape unit (natural boundary) "Sopka Luzanova”, 6.VII.2016, 1 ex.

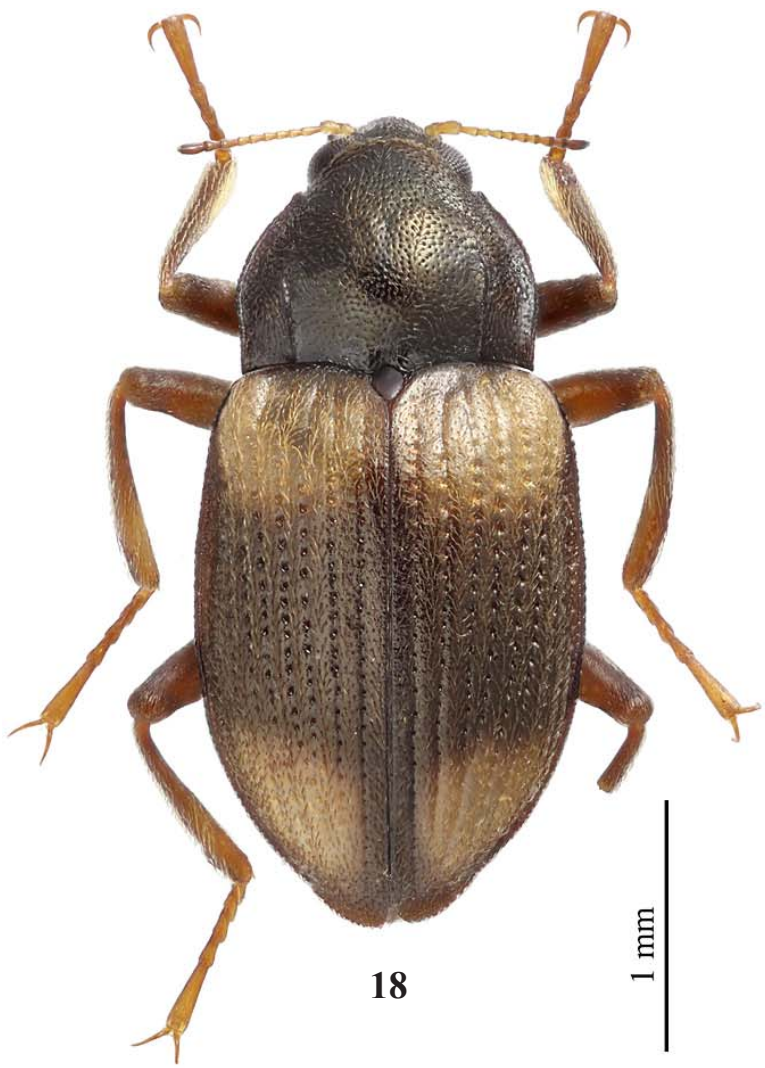

DISTRIBUTION. Widely distributed in Europe, North Africa, Central Asia, West Siberia; introduced to North America [Fikáček et al., 2015].

NOTE. The first record from Russian Far East and Primorsky Krai.

Sphaeridium scarabaeoides (Linnaeus, 1758)

MATERIAL EXAMINED. Primorsky Krai: Khasansky Distr., "Kedrovaya Pad" Nature Reserve, 3.V.2015, 2 exs.

DISTRIBUTION. Widely distributed in Europe, North Africa, Near and Central Asia, East and West Siberia, northeastern China, Russian Far East (Amur Oblast, Khabarovsky Krai and Primorsky Krai); introduced to the Afrotropical, Australian, and Nearctic regions [Shatrovskiy, 1989b; Ryndevich, 2001c; Fikáček et al., 2015].

\section{Family Elmidae Curtis, 1830}

Heterlimnius gapyeongensis (Jung, Kamite et Bae, 2011) Figs 18-20.

MATERIAL EXAMINED. Ussuriysk District, 2 km S Kaymanovka village, Komarovka River, $43.6270^{\circ} \mathrm{N} 132.2650^{\circ} \mathrm{E} 23$.VIII.2007, 1 ex. (K.V. Makarov), [deposited in Moscow Pedagogical State University, Moscow]; env. Ussuriysk, Kaymanovka village, Barsukovka River, $43.6437^{\circ} \mathrm{N} 132.2504^{\circ} \mathrm{E}, 18 . \mathrm{VI} .2020,1$ ex. (I.A. Zabaluev).

DISTRIBUTION. China (Liaoning), Nord and South Korea, Russian Far East (Primorsky Krai) [Jung et al., 2011; Jäch et al., 2016].

NOTE. Photographs of this species were published online by K.V. Makarov from Primorsky Krai (Khasansky District, env. Kedroviy, $10 \mathrm{~km}$ SES Barabash, $43.0833^{\circ} \mathrm{N} 131.5500^{\circ} \mathrm{E}$, 17-24.VII.2018, window trap, K. Makarov and A. Zaitzev leg., $10^{7}$ ), misidentified as Heterlimnius hasegawai Nomura, 1958 (https://www.zin.ru/animalia/coleoptera/rus/hethaskm.htm).

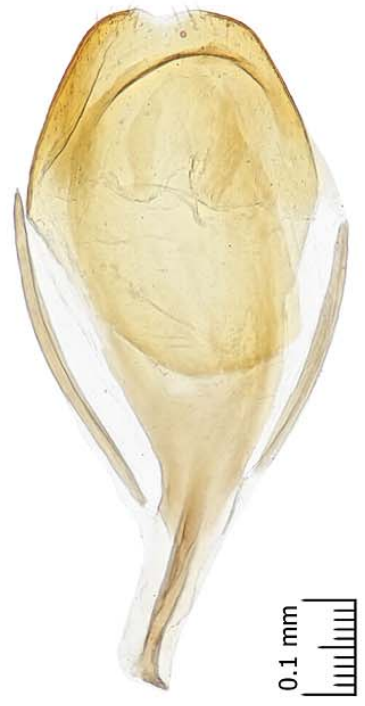

19

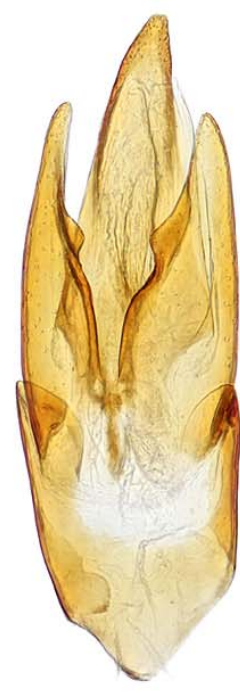

20
Figs 18-20. Heterlimnius gapyeongensis (Jung, Kamite et Bae, 2011) from Kaymanovka village: 18 - habitus, dorsal view; 19 ninth sternite; 20 - aedeagus. Photographs by K.V. Makarov.

Рис. 18-20. Heterlimnius gapyeongensis (Jung, Kamite et Bae, 2011) из с. Каймановка: 18 - габитус, сверху; 19 - девятый стернит; 20 - эдеагус. Фотографии К.В. Макарова. 
Thus, one species of Gyrinidae (Gyrinus sachalinensis), one species of Dytiscidae (Oreodytes mongolicus), and two species of Hydrophilidae (Sphaeridium bipustulatum and Sphaeridium marginatum) are recorded for the first time from Primorsky Krai. Three species (Oreodytes mongolicus, Sphaeridium bipustulatum, and Sphaeridium marginatum) are recorded for the first time from Russian Far East.

Acknowledgements. We are grateful to I.A. Zabaluev (Moscow, Russia), G.A. Nacharkin and D.E. Shcherbakov (Moscow, Russia), K.À. Ostapenko (Vladivostok, Russia) for material provided by them, P.N. Petrov (Moscow, Russia) for checking the identification of Ilybius anjae and valuable review of the manuscript, H. Fery (Berlin, Germany), G. Gustafson (Lawrence, USA) and J. Bergsten (Umea, Sweden) for providing some of the cited publications; V.M. Loktionov (Vladivostok, Russia) for processing photographs of the type specimens of Hydrocassis lucifer, and K.V. Makarov (Moscow, Russia) for photographs of Gyrinus sachalinensis, Enochrus japonicus, and Heterlimnius gapyeongensis. The work of A.S. Sazhnev and A.A. Prokin was carried out within the framework of a Russia State assignment no. 121051100109-1.

Competing interests. The authors declare no competing interests.

\section{References}

Baranowski R. 1985. Two new species of Cercyon Leach from Boreal Sweden // Scandinavian Entomolog. Vol.15. P.341-347.

Berlov E.Ya., Bervov O.E. 1996. [Catalogue of diving beetles (Coleoptera, Dytiscidae) of the Asian part of Russia] // Vestnik Irkutskoi Gosudarstvennoi Sel'skokhozyaistvennoi Akademii, Biological Issue. P.68-75 [in Russian].

Brinck P. 1943. Insecta, ex Sibiria meridionali et Mongolia, in itinere Orjan Olsen 1914 collecta. A. Coleoptera, a Fritz Jensen lecta. VIII. Haliplidae, Dytiscidae, Gyrinidae // Norsk Entomologisk Tidsskrift. Vol.6. P.154-161.

Enkhnasan D., Boldgiv B. 2019. Biogeography of predaceous diving beetles (Coleoptera, Dytiscidae) of Mongolia // ZooKeys. Vol.853. P.87-108. doi.org/10.3897/zookeys.853.33908

Fery H., Petrov P.N. 2014. Notes on the identity of Oreodytes okulovi Lafer 1988 (Coleoptera: Dytiscidae) // Linzer Biologische Beiträge. Vol.46. No.2. P.1241-1254.

Fikáček M., Angus R.B., Gentili E., Jia F., Minoshima Y.N., Prokin A., Przewoźny M., Ryndevich S.K. 2015. Family Hydrophilidae Latreille, 1802. // I. Löbl, D. Löbl (eds.). Catalogue of Palaearctic Coleoptera. Vol. 2. Revised and Updated Edition. LeidenBoston: Brill. P.37-76.

Fossen E.I., Ekrem T., Nilsson A.N., Bergsten J. 2016. Species delimitation in northern European water scavenger beetles of the genus Hydrobius (Coleoptera, Hydrophilidae) // ZooKeys. Vol.564: P.71-120. doi.org/10.3897/zookeys.564.6558.

Gramma V.N. 1980. [A new species of the genus Haliplus Latr. (Coleoptera, Haliplidae) from the Far East] // Entomologicheskoe Obozrenie. Vol.59. No.2. P.294-296 [in Russian, with English abstract].

Gusakov A.A. 2009. [Family Hydraenidae] // Storozhenko S.Yu (ed.). Nasekomye Lazovskogo zapovednika. Vladivostok: Dalnauka. P.114 [in Russian].

Hájek J. 2017a. Family Dytiscidae Leach, 1815 // I. Löbl, D. Löbl (eds). Catalogue of Palaearctic Coleoptera. Vol.1. Revised and updated edition. Archostemata - Myxophaga - Adephaga. Leiden-Boston: Brill. P.844-845.

Hájek J. 2017b. Family Noteridae C.G. Thomson, 1860 // I. Löbl, D. Löbl (eds.). Catalogue of Palaearctic Coleoptera. Vol.1. Revised and updated edition. Archostemata - Myxophaga - Adephaga. Leiden-Boston: Brill. P.846-914.

Hájek J., Fery H. 2017. Family Gyrinidae Latreille, 1810 // I. Löbl, D. Löbl (eds). Catalogue of Palaearctic Coleoptera. Vol.1.
Revised and updated edition. Archostemata - Myxophaga Adephaga. Leiden-Boston: Brill. P.22-29.

Hebauer F. 1994. The Crenitis of the Old World (Coleoptera, Hydrophilidae) // Acta Coleopterologica. Vol.10. No.2. P.3-40.

Hebauer F. 1995. Bekante und neue Hydrophiloidea aus Ostsibirien (Col.) // Entomologische Nachrichten und Berichte. Vol.39. P.29-36.

Hebauer F., Ryndevich S.K. 2005. New data on the distribution of Old World Hydrophilidae (Coleoptera) // Acta Coleopterologica. Vol.21. No.1. P.43-51.

Jäch M.A. 1988. Revisional notes on the Hydraena riparia species complex (Coleoptera: Hydraenidae) // Aquatic Insects. Vol.10. No.3. P.125-139.

Jäch M.A. 2015. Family Hydraenidae Mulsant, 1844 // Löbl I., Löbl D. (eds). Catalogue of Palaearctic Coleoptera. Vol.2. Revised and Updated Edition. Leiden-Boston: Brill. P.130-162.

Jäch M.A., Kodada J., Čiampor F. 2016. Family Elmidae Curtis, 1830 // Löbl I., Löbl D. (eds). Catalogue of Palaearctic Coleoptera, Vol. 3. Scarabaeoidea - Scirtoidea - Dascilloidea Buprestoidea - Byrrhoidea. Revised and updated edition. Leiden: Brill. P.591-603.

Jung S.W., Kamite Yu., Bae Y.J. 2011. Description of Optioservus gapyeongensis new species and Heterlimnius hasegawai (Nomura) (Coleoptera: Elmidae) new to Korea // Entomological Research. Vol.41. P.178-184.

Kobayashi T., Hayashi M., Kamite Yu., Sota T. 2021. Molecular phylogeny of Elmidae (Coleoptera: Byrrhoidea) with a focus on Japanese species: implications for intrafamilial classification // Systematic Entomology. P.1-13. DOI: 10.1111/syen.12499.

Lafer G.Sh. 1988. [New species of diving beetles (Coleoptera, Dytiscidae) from the Far East of the USSR] // Fauna, sistematika i biologiya presnovodnykh bespozvonochnykh. Vladivostok: DVO AN SSSR. P.52-59 [in Russian].

Lafer G.Sh. 1989. 7. [Fam. Dytiscidae - The Diving beetles] // Lehr P.A. (ed.). Opredelitel' nasekomykh Dal'nego Vostoka SSSR. Vol.3. No.1. Lenindrad: Nauka Publ. P.229-253 [in Russian].

Lafer G.Sh. 1992. [Corrections and clarifications to the book "Keys to the Insects of the Far East of the USSR, Vol. 1, part 1" (1989 yr)] // Lehr P.A. (ed.). Opredelitel' nasekomykh Dal'nego Vostoka SSSR. Vol.3. No.2. Sankt-Peterburg: Nauka Publ. P.666702 [in Russian].

Lafer G.Sh. 1996. Fam. Haliplidae. Dopolneniye 1. [Addition 1]// Lehr P.A. (ed.). Opredelitel' nasekomykh Dal'nego Vostoka Rossii. Vol.3. No.3. Vladivostok: Dal'nauka. P.408 [in Russian].

Larson D.J., Alarie Y., Roughley R.E. 2000. Predaceous diving beetles (Coleoptera: Dytiscidae) of the Nearctic region, with emphasis on the fauna of Canada and Alaska. Ottawa: NRC Research Press. 982 pp.

Maslovskaia O., Kopeva A., Petrova E., Ivanova O., Khrapko O. 2020. Development of ecotourism infrastructure at protected areas of Primorsky Krai // IOP Conf. Series: Materials Science and Engineering. Vol.890. P.1-6. doi:10.1088/1757-899X/890/ $1 / 012002$.

Nilsson A.N. 1994. Two new East Palearctic Agabus species of the adpressus- and confinis-groups (Coleoptera, Dytiscidae) // Entomologica Fennica. Vol.5. P.169-175.

Nilsson A.N. 1999. Description of a new East Palearctic species of Ilybius Erichson previously mixed up with I. poppiusi Zaitzev (Coleoptera: Dytiscidae) // Koleopterologische Rundschau, Vol.69. P.33-40.

Nilsson A.N., Minakawa N., Oberg P.B.H. 1997. The diving beetles of the Kuril Archipelago in the Far East of Russia (Coleoptera: Dytiscidae) // Beiträge zur Entomologie. Vol.47. No.2. P.365-376.

Nilsson A.N., Kholin S.K. 1994. The diving beetles (Coleoptera, Dytiscidae) of Sakhalin - an annotated checklist // Entomologisk Tidskrift. Vol.115. P.143-156.

Nilsson A.N., Kholin S.K., Minakawa N. 1999. The diving beetles of Kamchatka, with additional records from Sakhalin and the Kuril Islands // Beiträge zur Entomologie. Vol.49. No.1. P.107-131.

Nilsson A.N., Lundmark M., Kholin S.K., Minakawa N. 2001. A faunistic review of the Gyrinus species of the Far East of Russia // Koleopterologische Rundschau. Vol.71. P.27-35. 
Nilsson A.N., Sundukov Yu.N. 2009a. [Family Dytiscidae] // Storozhenko S.Yu (ed.). Nasekomye Lazovskogo zapovednika. Vladivostok: Dalnauka. P.87-88 [in Russian].

Nilsson A.N., Sundukov Yu.N. 2009b. [Family Haliplidae] // Storozhenko S.Yu(ed.). Nasekomye Lazovskogo zapovednika. Vladivostok: Dalnauka. P.86 [in Russian].

Nilsson A.N., Sundukov Yu.N. 2009c. [Family Noteridae] // Storozhenko S.Yu(ed.). Nasekomye Lazovskogo zapovednika. Vladivostok: Dalnauka. P.87 [in Russian].

Ôhara M., Jia F.-L. 2006. Terrestrial Hydrophilid Beetles of the Kuril Archipelago // Biodiversity and Biogeography of the Kuril Islands and Sakhalin. No.2. P.129-150.

Palatov D.M. 2014. [New data on the benthic macroinvertebrate fauna in fresh waters of Kunashir Island] // Chteniya pamyati Vladimira Yakovlevicha Levanidova. Vol.6. P.509-522 [in Russian].

Pimenova E.A. 2016. [Hydrology and hydrography, Vascular plants] // Pimenova E.A. (ed.). Plants, fungi and lichens of the SikhoteAlin Reserve. Vladivostok: Dalnauka P.11-14, 172-365 [in Russian, with English abstract].

Prokin A.A. 2009. [Family Hydrophilidae] // Storozhenko S.Yu (ed.). Nasekomye Lazovskogo zapovednika. Vladivostok: Dalnauka. P.110-112 [in Russian].

Prokin A.A. 2010. [New data on distribution of Hydrochidae and Hydrophilidae (Coleoptera) in Russia and adjacent countries. Problems of water entomology in Russia and adjacent countries] // Materialy X trikhopterologicheskogo simpoziuma i IV Vserossiyskogo simpoziuma po amfibioticheskim i vodnym nasekomym. Vladikavkaz: Severo-Osetinsk. Gosud. Univ. Publ. House. P.74-78 [in Russian].

Prokin A.A., Chuluunbaatar G., Angus R.B., Jäch M.A., Petrov P.N., Ryndevich S.K., Byambanyam E., Sazhnev A.S., Shaverdo H. 2020. New records of water beetles (Coleoptera: Gyrinidae, Haliplidae, Noteridae, Dytiscidae, Helophoridae, Hydrophilidae, Hydraenidae) and shore beetles (Coleoptera: Heteroceridae) of Mongolia // Aquatic Insects. Vol.41. No.1. P.1-44. doi.org/10.1080/01650424.2019.1651870.

Ryndevich S.K. 2001a. New data on Holarctic and Oriental Spercheidae and Hydrophilidae (Coleoptera: Hydrophiloidea) // Euroasian Entomological Journal. Vol.10. No.3. 337-340.

Ryndevich S.K. 2001b. On identification of species of the Cercyon dux group (Coleoptera: Hydrophilidae) // Zoosystematica Rossica. Vol.1. No.1. P.79-83.

Ryndevich S.K. 2001c. Review of genus Sphaeridium Fabricius, 1775 (Coleoptera: Hydrophilidae) of fauna of Belarus and adjacent regions // Byulleten' Moskovskogo Obshchestva Ispytatelei Prirody. Seriya Biologicheskaya. Vol.106. No.3. P.22-29.

Ryndevich S.K. 2003. Some records of Dytiscidae, Helophoridae, Hydrochidae, Hydrophilidae and Hydraenidae in Russia and other regions // Latissimus. Vol.16. P.17-20.

Ryndevich S.K. 2011. New data on Holarctic and Oriental Spercheidae and Hydrophilidae (Coleoptera: Hydrophiloidea) // Euroasian Entomological Journal. Vol.10. No.3. P.337-340.

Ryndevich S.K. 2016. Species of genus Hydrobius (Coleoptera: Hydrophilidae) from Belarus // Baranovichi State University
Herald, Series: Biological Sciences, Agricultural Sciences. Vol.4. P.63-71.

Ryndevich S.K., Angus R.B. 2020. Redescription of Hydrobius pauper (Coleoptera: Hydrophilidae), with a key to the Eurasian species of the genus Hydrobius // Zoosystematica Rossica. Vol.29. No.1. P.77-86.

Ryndevich S.K., Fikáček M. 2013. Faunistic and zoogeographic notes on hydrophiloid beetles from the Palaearctic region // Baranovichi State University Herald, Series: Biological Sciences, Agricultural Sciences. Vol.1. P.32-37.

Ryndevich S.K., Prokin A.A. 2017. Two new species of Cercyon (Clinocercyon) from Russian Far East (Coleoptera: Hydrophilidae) // Zootaxa. Vol.4300. No.1. P.125-134. doi.org/10.11646/ zootaxa.4300.1.7.

Sazhnev A.S. 2018. New records of water beetles (Coleoptera: Helophoridae, Hydrophilidae, Hydraenidae) from Commander Islands // Far Eastern Entomologist. Vol.365. P.26-30. doi.org/ $10.25221 /$ fee. 365.2 .

Shatrovsky A.G. 1986. [Water beetles of the genus Hydrochara (Coleoptera, Hydrophilidae) of the USSR fauna] // Vestnik Zoologii. Vol.4. P.29-33 [in Russian, with English abstract].

Shatrovskiy A.G. 1989a. [11. Fam. Hydraenidae (Limnebiidae)] // Lehr P.A. (ed.). Opredelitel' nasekomykh Dal'nego Vostoka SSSR. Vol.3. No.1. Lenindrad: Nauka Publ. P.260-264 [in Russian].

Shatrovskiy A.G. 1989b. [12. Fam. Hydrophilidae] // Lehr P.A. (ed.). Opredelitel' nasekomykh Dal'nego Vostoka SSSR. Vol.3. No.1. Lenindrad: Nauka Publ. P.264-293 [in Russian].

Shatrovskij A.G. 1992. [New and little known Hydrophiloidea (Coleoptera) from Southern Primorye Territory and adjacent regions] // Entomologicheskoe Obozrenie. Vol.71. P.359-371 [in Russian, with English title].

Schödl S. 1991. Revision der gattung Berosus Leach 1.Teil: Die paläarktischen arten der untergattung Enoplurus (Coleoptera: Hydrophilidae)//Koleopterologische Rundschau. Vol.61.P.111135.

Schödl S. 1993. Revision der gattung Berosus Leach. 3. Teil: Die palaarktischen und orientalischen arten der untergattung Berosus s. str. (Coleoptera: Hydrophilidae) // Koleopterologische Rundschau. Vol.63. P.189-233.

Smetana A. 1978. Revision of the subfamily Sphaeridiinae of America north of Mexico (Coleoptera: Hydrophilidae) // Memoirs of the Entomological Society of Canada. Vol.105. P.1-292.

Smetana A. 1980. Revision of the genus Hydrochara Berth, (Coleoptera: Hydrophilidae) // Memoris of the Entomological Society of Canada. Vol.111. P.1-100.

Smetana A. 1988. Review of the family Hydrophilidae of Canada and Alaska // Memoirs of the Entomological Society of Canada. Vol.120. P.3-316.

van Vondel B.J. 2017. Family Haliplidae Aubé, 1836 // I. Löbl, D. Löbl (Eds.). Catalogue of Palaearctic Coleoptera. Vol. 1. Archostemata - Myxophaga - Adephaga. Revised and updated edition. Leiden: Brill. P.838-843.

Zaitzev Ph.A. 1953 [Dytiscoids and Gyrinids] // Fauna SSSR. Vyp.58. Nasekomye zhestkokrylye. T.4. Novaya seriya. Moscow-Leningrad: AN SSSR Publ. 377 pp. [In Russian] 